\title{
Gastric duplication cyst in an adult with autoimmune hemolytic anemia: a case report and review of the literature
}

\author{
Amal Bennani ${ }^{1,4^{*}}$, A. Miry ${ }^{1}$, I. Kamaoui ${ }^{2}$ and T. Harroudi ${ }^{3}$
}

\begin{abstract}
Background: Gastric duplication cysts are uncommon congenital anomalies found primarily in children and rarely seen in the adult population. Accurate diagnosis of cysts before resection is difficult even using the most advanced imaging techniques.

Case presentation: In this report, we describe a 28-year-old Moroccan patient with a history of autoimmune hemolytic anemia who presented with an asymptomatic abdominal cystic mass detected during abdominal computed tomography performed before splenectomy. Magnetic resonance imaging performed for accurate characterization showed a high-signal-intensity cystic mass on T2-weighted images, located between the patient's stomach and spleen. The patient underwent a complete cyst resection during exploratory laparotomy. The histological examination showed a cyst lined by three different epithelia with bundles of smooth muscle, which suggested a gastric duplication cyst.

Conclusions: We report a case of gastric cyst duplication in an adult with autoimmune hemolytic anemia, and we discuss this rare association, radiological findings, and the unique histological findings of this case.
\end{abstract}

Keywords: Duplication, Abdominal cyst, Gastric, Epithelium

\section{Background}

A gastrointestinal duplication is defined as a spherical cystic structure lined by a mucous membrane with smooth muscle in its wall [1]. It may occur anywhere along the mesenteric side of the gastrointestinal tract, most commonly in the ileum (35\%). Gastric duplication cysts (GDCs) are a rare finding and account for only $2-9 \%$ of all gastrointestinal duplications [2]. Although most cases are diagnosed within the first year of life, a few cases have been diagnosed in adulthood [3]. The diagnosis of these cysts using all advanced imaging techniques before resection is difficult. We report a rare case of a patient with GDC with the aim of shedding more light on this uncommon developmental anomaly and on the fact that it should be considered in the differential diagnosis of abdominal cystic masses in adulthood, although the clinical and radiological findings are somewhat nonspecific. The current case is unique because it has an unusual histological finding and it

\footnotetext{
* Correspondence: bennaniamal@gmail.com

${ }^{1}$ Department of Pathology, Mohamed I University, 30050 Oujda, Morocco

${ }^{4}$ Assaada, Bp 6210, 60020 Oujda, Morocco

Full list of author information is available at the end of the article
}

is associated with an autoimmune hemolytic anemia, which allowed us to discuss a possible morbid association.

\section{Case presentation}

We present a case of a 28-year-old white man with an 8-month history of clinical symptoms of anemia, such as fatigue and breathlessness, without any abdominal complaint. He had autoimmune hemolytic anemia treated by corticosteroids for more than 6 months but with a relapse of disease after steroid remission. For this reason, he was a candidate for a splenectomy. His occupation is a student. He did not smoke tobacco or consume alcohol, and he was taking prednisone, bisphosphonates, vitamin $\mathrm{D}$, and calcium. No other immune disorders were found. On admission, his blood pressure was $90 / 70 \mathrm{mmhg}$, his heart rate was 70 beats/minute, and his body temperature was $36^{\circ} \mathrm{C}$. Physical examination of the patient revealed a severe pale conjunctiva and icteric sclera with no evidence of abdominal mass or other physical abnormalities. Laboratory analysis results on admission are shown in Table 1.

Abdominal computed tomography (CT) performed before the splenectomy showed a large cystic mass 
between the stomach and the spleen with no evidence of communication with the stomach or pancreas. Endoscopic ultrasound (EUS) showed a cystic mass located along the greater curvature with no mucosal abnormality or communication with the gastric lumen. Magnetic resonance imaging performed for more characterization showed a cystic mass between the stomach and spleen with a high signal intensity on T2-weighted fat-saturated magnetic resonance images (Fig. 1) and peripheral enhancement after gadolinium injection (Fig. 2). At this stage, the differential diagnoses included hydatid cyst, mesenteric cyst, and pancreatic pseudocyst.

The patient underwent exploratory laparotomy with complete cyst resection and splenectomy. At gross examination, the cystic mass, measuring $8 \times 5.5 \times 4$ $\mathrm{cm}$, was well-circumscribed, unilocular, and filled with a mucoid yellowish fluid. Histologically, the cystic mass was lined by gastric epithelium with pyloric glands (Fig. 3), transitioning focally into squamous epithelium (Fig. 4) and in some areas into pseudostratified columnar ciliated epithelium (Fig. 5). A small part of the mucosa-like wall had ulceration accompanied by inflammation (Fig. 6). There was no evidence of cartilaginous tissue in the wall. There were also bundles of smooth muscle in the wall. This finding suggested a GDC .

The histological examination of splenectomy showed pronounced cord congestion with reactive follicular hyperplasia and increased deposition of hemosiderin. After 10 months, there were no signs of local complication, and the patient was successfully weaned from steroids and had complete resolution of hemolytic anemia with negative direct antiglobulin tests.

Table 1 Laboratory data on admission

\begin{tabular}{|c|c|}
\hline Test & Results \\
\hline \multicolumn{2}{|l|}{ Hematology } \\
\hline WBC & $11.5 \times 10^{9} / \mathrm{L}$ \\
\hline $\mathrm{RBC}$ & $2.2 \times 10^{12} / L$ \\
\hline $\mathrm{Hb}$ & $6.8 \mathrm{~g} / \mathrm{L}$ \\
\hline Hct & $24.3 \%$ \\
\hline MCV & $110 \mathrm{fl}$ \\
\hline Plt & $400 \times 10^{9} / \mathrm{L}$ \\
\hline $\mathrm{RC}$ & $18 \%$ \\
\hline \multicolumn{2}{|l|}{ Biochemistry } \\
\hline TP & $80 \mathrm{~g} / \mathrm{L}$ \\
\hline CRP & $4 \mathrm{mg} / \mathrm{L}$ \\
\hline Urea & $0.3 \mathrm{~g} / \mathrm{L}$ \\
\hline Creatinine & $7 \mathrm{mg} / \mathrm{L}$ \\
\hline
\end{tabular}

Abbreviations: $\mathrm{Cr}$ Creatinine, CRP C-reactive protein, $\mathrm{Hb}$ Hemoglobin, $\mathrm{Hct}$ Hematocrit, MCV Mean corpuscular volume, Plt Platelets, RBC Red blood cells, $R C$ Reticulocyte count, TP Total protein, WBC White blood cells

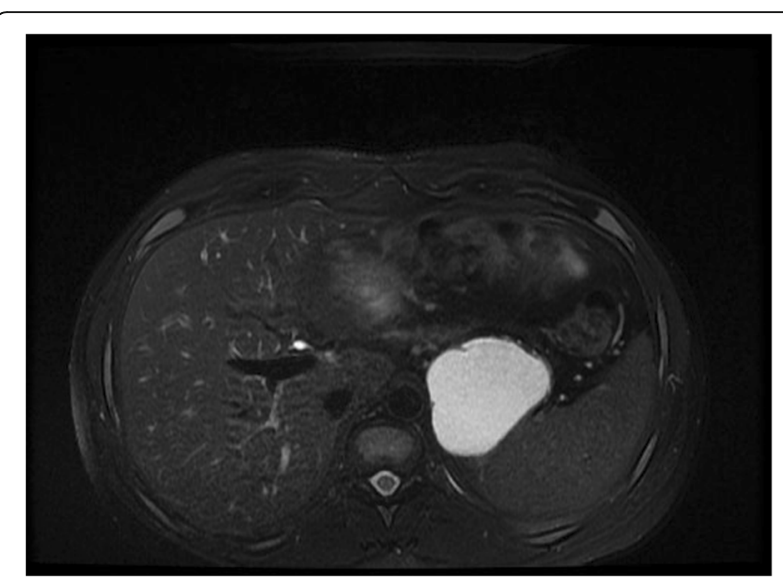

Fig. 1 T2-weighted fat-saturated magnetic resonance image shows a high-signal-intensity cystic mass between stomach and spleen

\section{Discussion}

In this report, we present a rare association of a hemolytic autoimmune anemia and an asymptomatic GDC in a 28-year-old man. This association has not been reported in the literature so far. We also describe a unique histological morphology of this cyst, which was lined by three different types of epithelium. Duplications of the alimentary tract are rare and occur in 1 in 4500 births [4]. Gastric duplications are infrequent and constitute $8 \%$ of all duplications. Duplication cysts of the ileum are usually located on the mesenteric border, whereas the usual location for GDCs is along the greater curvature [5], as in our patient. It is believed that they are congenital developmental alterations within the gastrointestinal tract. Many theories exist for the development of these lesions, including a persistent

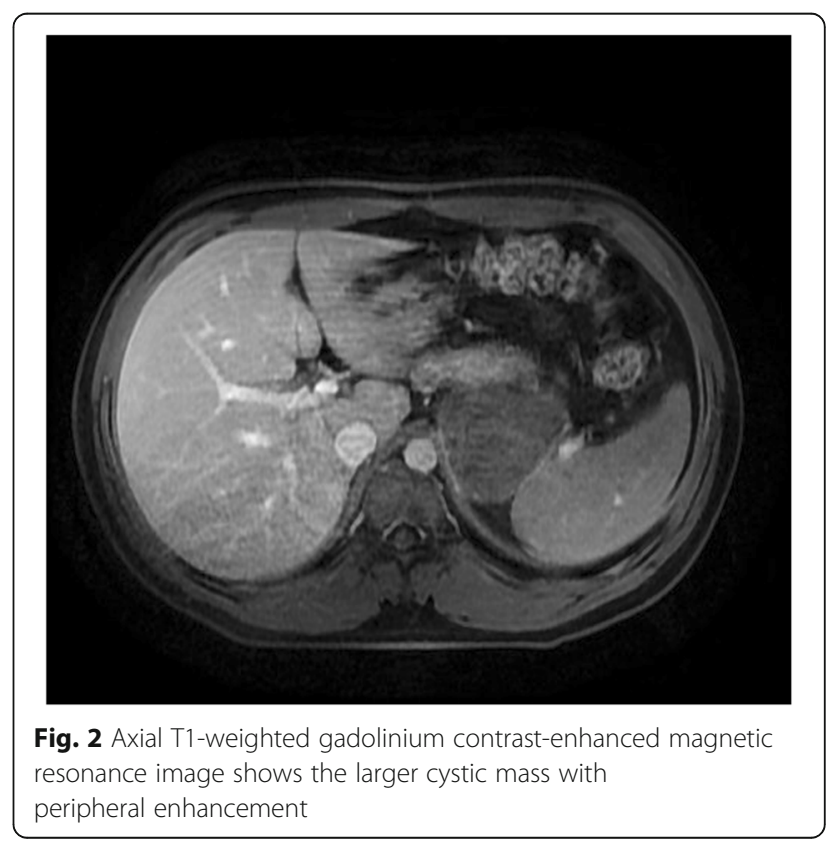




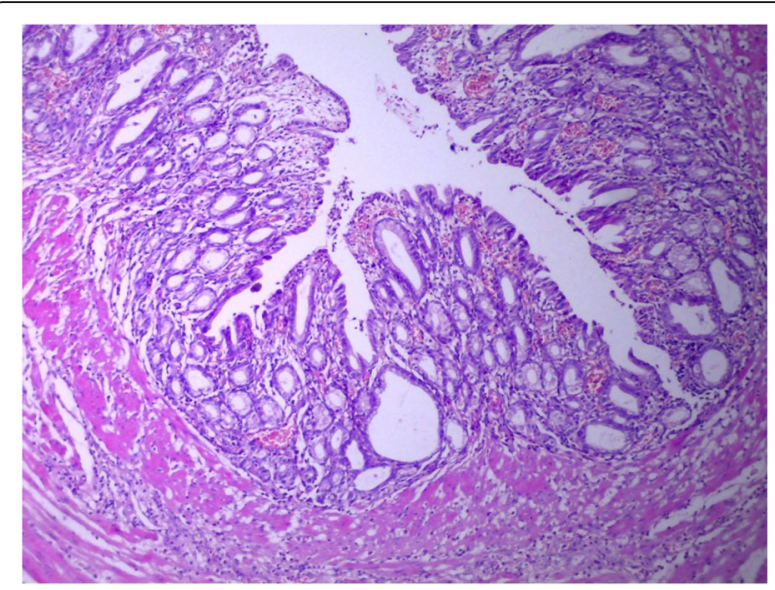

Fig. 3 The cystic mass was lined by gastric epithelium with pyloric glands, and bundles of smooth muscle can be seen in the wall

embryological diverticulum, aberrant recanalization of the alimentary tract, partial twinning, and in utero ischemic events [6]. The majority of cases are diagnosed in the pediatric population within the first 3 months of life and rarely in patients over 12 years of age [7].

There are two types of duplication: tubular when the lumen is contiguous with stomach lumen and cystic when the lumen is not contiguous with stomach lumen [8]. More than $80 \%$ of gastric duplications are cystic and do not communicate with the stomach lumen. The epithelial lining of duplication may be histologically similar to the segment to which the cyst is topographically related, or it may include, in a minority of cases, lining from another segment of the alimentary tract or cellular components that do not resemble those naturally occurring in the gastrointestinal tract, such as ciliated and respiratory-type epithelium and cartilage.

Our patient exhibited a unique histological finding: The lining epithelium was composed of both squamous and

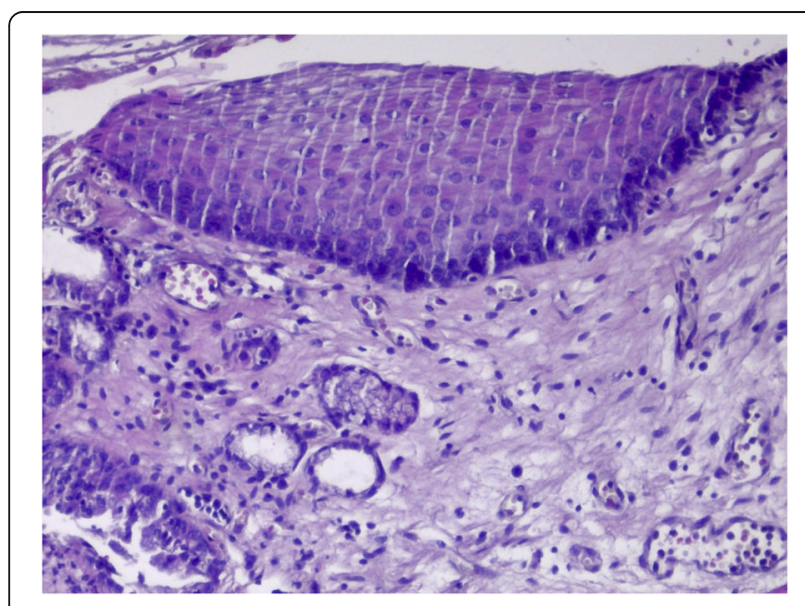

Fig. 4 Squamous epithelium

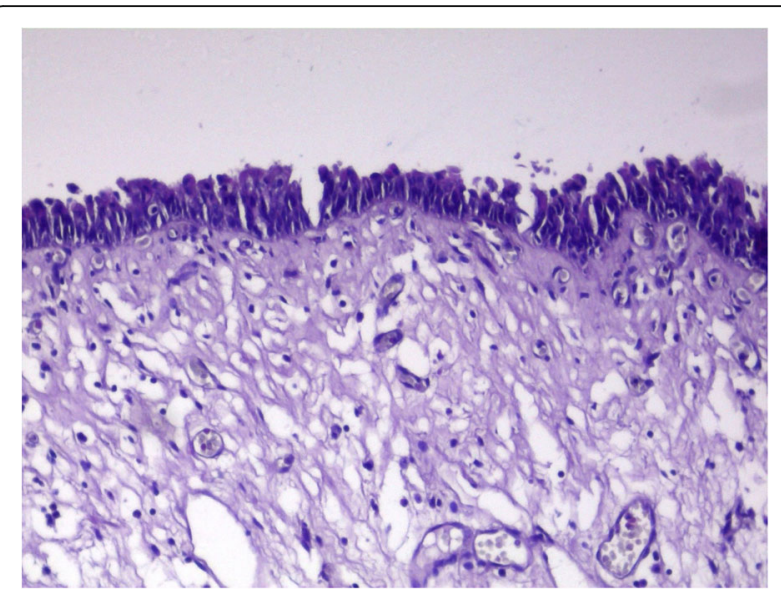

Fig. 5 Respiratory pseudostratified epithelium

respiratory mucosa with the gastric epithelium, which has not been reported so far in the same cyst. The cyst lining may undergo erosions, ulceration, and regenerative changes due to increased fluid pressure. These changes may lead to bleeding into the cyst or perforation into the peritoneal cavity. These cysts are usually asymptomatic and are diagnosed incidentally, as observed in the cases in this report. However, in some cases, they have no specific symptoms, such as epigastralgia, vomiting, anemia, or recurrent episodes of pancreatitis. They may also manifest as complications such as gastric outlet obstruction, gastric perforation, and carcinoma arising in the cyst [9].

Our patient's case represents a very rare association between GDCs and hemolytic autoimmune anemia. Hemolytic anemia is commonly associated with lymphoproliferative neoplasms, drugs, connective tissue diseases, and infections and are very rarely reported in association with ovarian dermoid cysts and mesenteric dermoid cysts.

There is only one reported case of a rare association between an enteric duplication cyst and autoimmune

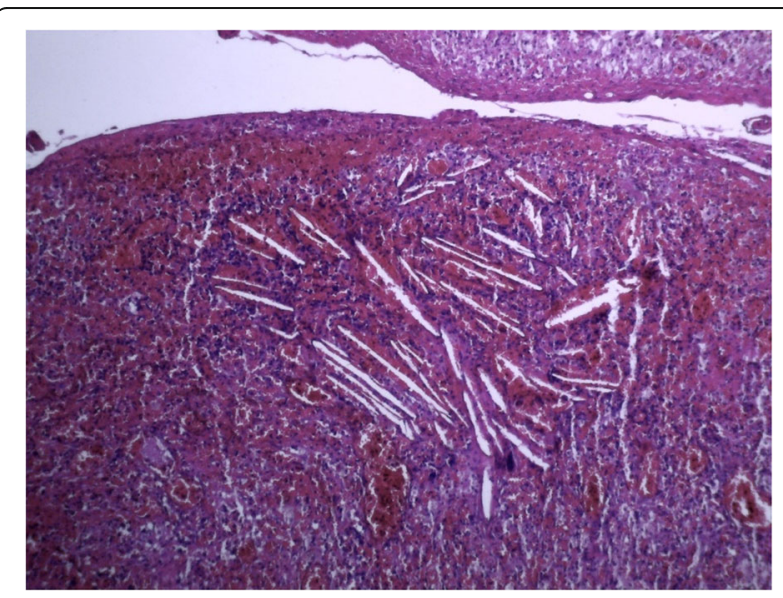

Fig. 6 Area of ulceration and inflammation 
hemolytic anemia. In that case, there was an adenocarcinoma arising in the cyst, which refers to a paraneoplastic autoimmune hemolytic anemia [10]. In our patient, the microscopic study of the resected cyst did not show malignancy. Furthermore, because we performed a splenectomy at the same time and the hemolytic anemia resolution was obtained after surgery, we cannot give a definite conclusion on the underlying etiology. However, there is a strong possibility of association between the cyst and anemia. This case presented a real diagnostic conundrum. The recognition of this association requires further studies and more reported cases.

Although most of the diagnoses are made postoperatively, several imaging modalities have provided some informative findings. CT and EUS are very useful in identifying GDCs [9]. Classically, contrast-enhanced CT typically demonstrates GDC as a thick-walled cystic lesion with enhancement of the inner lining [11]. Calcification is occasionally observed.

EUS is useful in distinguishing between the intramural and extramural lesions of the stomach. Compared with $\mathrm{CT}$, magnetic resonance imaging can provide additional information about cyst content. However, the nature of the fluid in the GDC is different in each case according to bleeding, chronic inflammation, or infection. Treatment is complete excision of the cyst to avoid the risk of possible complications such as perforation, hemorrhage, and the risk of neoplastic transformation $[12,13]$.

\section{Conclusions}

A GDC is a rare congenital anomaly that is difficult to diagnose before complete resection. It should be considered in the differential diagnosis of abdominal cystic lesions. Its association with hemolytic autoimmune anemia raises the possibility of a morbid association and requires more reported cases.

\section{Abbreviations \\ Cr: Creatinine; CRP: C-reactive protein; CT: Computed tomography; \\ GDC: Gastric duplication cyst; EUS: Endoscopic ultrasound; Hb: Hemoglobin; Hct: Hematocrit; MCV: Mean corpuscular volume; Plt: Platelets; RBC: Red blood cells; RC: Reticulocyte count; TP: Total protein; WBC: White blood cells}

\section{Acknowledgements}

Not applicable.

Funding

Not applicable.

\section{Availability of data and materials}

The authors agree to make the raw data and materials described in our report freely available.

\section{Authors' contributions}

$A B$ wrote the article. AM arranged the figures. IK provided some of the radiological images and commented on the manuscript. TH is the surgeon who treated the patient. All authors read and approved the final manuscript.

Ethics approval and consent to participate Not applicable.

\section{Consent for publication}

Written informed consent was obtained from the patient for publication of this case report and any accompanying images. A copy of the written consent is available for review by the Editor-in-Chief of this journal.

\section{Competing interests}

The authors declare that they have no competing interests.

\section{Publisher's Note}

Springer Nature remains neutral with regard to jurisdictional claims in published maps and institutional affiliations.

\section{Author details \\ 'Department of Pathology, Mohamed I University, 30050 Oujda, Morocco. ${ }^{2}$ Department of Radiology, Mohamed I University, 30050 Oujda, Morocco. ${ }^{3}$ Department of Surgical Oncology, Mohamed I University, 30050 Oujda, Morocco. ${ }^{4}$ Assaada, Bp 6210, 60020 Oujda, Morocco.}

Received: 27 March 2018 Accepted: 26 October 2018

Published online: 26 December 2018

References

1. Doepker MP, Ahmad SA. Gastric duplication cyst: a rare entity. J Surg Case Rep. 2016;2016(5):rjw073.

2. Scatizzi M, et al. Gastric duplication cyst in an adult: case report. In Vivo. 2005;19(6):975-8.

3. Houschek V, et al. Gastric duplication cyst: a rare endosonographic finding in an adult. Scand J Gastroenterol. 2005;40(9):1129-31.

4. Lewis PL, Holder T, Feldman M. Duplication of the stomach: report of a case and review of the English literature. Arch Surg. 1961;82(4):634-40.

5. Laraja RD, et al. Foregut duplication cyst: a report of a case. Am Surg. 1995; 61(9):840-1.

6. Bonacci JL, Schlatter MG. Gastric duplication cyst: a unique presentation. J Pediatr Surg. 2008;43(6):1203-5.

7. Takazawa $\mathrm{S}$, et al. Laparoscopic partial gastrectomy of a huge gastric duplication cyst in an infant. Nagoya J Med Sci. 2015;77(1-2):291-6.

8. Murakami S, et al. Foregut duplication cyst of the stomach with pseudostratified columnar ciliated epithelium. Pathol Int. 2008;58(3):187-90.

9. D'Journo X, et al. Gastric duplication in an adult mimicking mucinous cystadenoma of the pancreas. J Clin Pathol. 2004;57(11):1215-8.

10. Sreedhar A, Nair R, Scialla W. Paraneoplastic auto-immune hemolytic anemia: an unusual sequela of enteric duplication cyst. Anticancer Res. 2018;38(1):509-12.

11. Maeda $\mathrm{H}$, et al. Diagnostic challenge to distinguish gastric duplication cyst from pancreatic cystic lesions in adult. Intern Med. 2007;46(14):1101-4.

12. Mardi K, Kaushal V, Gupta S. Foregut duplication cysts of stomach masquerading as leiomyoma. Indian J Pathol Microbiol. 2010;53(4):829-30.

13. Johnston J, et al. Gastric duplication cysts expressing carcinoembryonic antigen mimicking cystic pancreatic neoplasms in two adults. Am Surg. 2008;74(1):91-4. 